
\title{
25 Research Square \\ Long-Term Electrically Stable Silver Nanowire Composite Transparent Electrode Under High Current Density
}

\section{Kaiqing Wang}

Fudan University https://orcid.org/0000-0003-2581-4441

Yunxia Jin

Fudan University

Fei Xiao ( $\square$ feixiao@fudan.edu.cn )

Fudan University

\section{Research Article}

Keywords: silver nanowire, chitosan-ascorbic acid, composite transparent electrode, long-term electrical stability

Posted Date: June 4th, 2021

DOl: https://doi.org/10.21203/rs.3.rs-287036/v1

License: (c) (i) This work is licensed under a Creative Commons Attribution 4.0 International License. Read Full License

Version of Record: A version of this preprint was published at Journal of Materials Science: Materials in Electronics on July 28th, 2021. See the published version at https://doi.org/10.1007/s10854-021-063864. 


\section{Abstract}

Silver nanowire (AgNW) network has been employed to many electronic devices as transparent electrode. However, the poor electrical stability under current has been seriously holding its practical application, and we still lack long-term electrically stable AgNW system to study the underlying fundamental of electrical failure. In this work, the electrical performance and failure mechanism of chitosan-ascorbic acid (Chi-AsA)/AgNW composite under current stress were thoroughly studied. The composite electrode maintained stability above $24000 \mathrm{~h}$ under high current density of $100 \mathrm{~mA} \mathrm{~cm}^{-1}$. The main failure in AgNW composite is found to be a wave break perpendicular to the current rather than traditional uniform degradation across AgNW networks. More interestingly, the AgNWs in failed composite electrode kept their original smooth morphology excepting the crack area, while the AgNWs in pristine networks degraded to nanoparticles or became disconnected everywhere. The patterned AgNW composite in microscale exhibits similar long lifetime in resisting current stress as the bulk composite film. The effect of over-coating position, electrical stress, temperature and over-coating materials on the electrical stability were studied. The over-coating layer of Chi-AsA is proven to suppress the silver atoms from migration, reduce the concentrated Joule heating at junctions, and inhibit the corrosion. The Chi-AsA/AgNW composite enables electrically stable transparent conductor for next-generation optoelectronics, and the mechanism investigation may provide effective means of preparing electrically stable AgNW systems.

\section{Introduction}

Transparent electrode constitutes crucial component of various optoelectronic devices. The main transparent conductive materials have been metal oxides for decades, but alternative flexible film electrodes have attracted intense attention because of emerging flexible optoelectronics. Particularly, silver nanowire (AgNW) networks can be fabricated with easy solution process, and exhibit comparable or even better opto-electronic performance compared with traditional indium tin oxide (ITO), showing advantages to other candidates [1]. It has been efficiently integrated into various devices such as solar cells [2], organic light-emitting diodes (OLEDs) [3], film heaters [4], touch screens [5], sensors [6], and energy electronics [7-8].

Despite the attractive application in lab scale, the AgNW transparent electrode has not been widely considered in industry because of the instability, especially the poor electrical stability. For example, the lifetime of AgNW film with sheet resistance of $12 \Omega \mathrm{sq}^{-1}$ was only 18 days at $17 \mathrm{~mA} \mathrm{~cm}{ }^{-2}$ [9], and sharply reduced to several hours under higher current density of $125 \mathrm{~mA} \mathrm{~cm}^{-2}$ [10]. However, the optoelectronics require service life of at least years under working state, which means the current is inevitable to flow through the transparent electrode. For example, the film heaters request current density of $10 \sim 30 \mathrm{~mA}$ $\mathrm{cm}^{-2}$ to achieve saturation temperature above $100^{\circ} \mathrm{C}$, and $\sim 100 \mathrm{~mA} \mathrm{~cm}^{-2}$ to higher than $200{ }^{\circ} \mathrm{C}[4,11-13]$. The short-circuit current density in OPV can reach $20 \mathrm{~mA} \mathrm{~cm}^{-2}$ [14-18] or above; and 10 50 $\mathrm{mA} \mathrm{cm}^{-2}$ is required for OLEDs to obtain the maximum efficiency, which exceeds $100 \mathrm{~mA} \mathrm{~cm}{ }^{-2}$ at the upper test bias 
voltage $[10,19-20]$. It is obviously that the poor electrical stability of AgNWs seriously cut down the lifetime of optoelectronic devices.

Unlike the continuous flat film, e.g. the traditional metal oxide, AgNW transparent electrode is composed of randomly interconnected silver nanowires with high aspect ratio of $\sim 1000$, which brings additionally new challenging problems. First, every single silver nanowire bears much higher current density compared to that loaded to the whole film. For example, a current density of $150 \mathrm{~mA} \mathrm{~cm}^{-2}$ on the AgNW network film turns out to an extremely high current density of $5 \times 10^{6} \mathrm{~A} \mathrm{~cm}^{-2}$ on individual nanowire [10]. Second, the Joule heating caused by current in cross-linked junctions between nanowires is quite strong due to the high contact resistance, which tends to melt the nanowires to droplets in junctions followed with capillary or Rayleigh instability [9-10, 21-22]. Third, the deposition of AgNW networks in nanoscale is not homogeneous, and may cause the non-uniform current flow, resulting in local hotspots and local failures [22-23]. Forth, the surface of pristine AgNW is prone to be corroded with sulfides, oxygen, moisture or other chemicals in ambient environment [24-26]. Last, the synergistic effect of electrical stress, Joule heating, and corrosion may speed up the failure of AgNW networks. Therefore, it has been a great challenge to improve electrical stability of AgNW networks.

Silver nanowire composites with passivation layer on the top or with mixed inert materials have been explored to slow down the nanowire degradation under current flow. Generally there are two main strategies. One is the introduction of solution processed carbon nanomaterials or conductive polymer. For example, graphene oxide (GO), reduced graphene oxide ( $\mathrm{rGO}$ ) obtained from chemical reaction, carbon nanotube (CNT), single wall carbon nanotube (SWCNT), or neutral poly(3,4-ethylenedioxythiophene): poly(styrenesulfonate) (PEDOT:PSS), was deposited on the top of AgNW networks [27-33]; SWCNT was mixed with AgNW solution to prepare composite electrodes [34-35]; GO was inserted into the multilayer AgNW networks [36]. The composites demonstrated better stability compared to pristine AgNW networks under current stress. The best result comes from the rGO/AgNW composite film with a lifetime of 12 days at $20 \mathrm{~mA} \mathrm{~cm}^{-1}$ [29]. However, the GO or CNT formed a network with gap rather than a dense passivation layer on the top, and impurities and inorganic ions in GO and CNT raw materials are inevitable and accelerate the failure somehow. Another strategy is the physical deposition of a fully covered layer of dense inorganic oxides on the top of AgNWs. For example, zinc oxide $(\mathrm{ZnO})$ or titanium dioxide $\left(\mathrm{TiO}_{2}\right)$ was deposited on individual nanowires through atomic layer deposition process [10, 37-40]. Five $\mathrm{nm}$ of $\mathrm{TiO}_{2}$ layer stabilized the $\mathrm{AgNW}$ network that sustained voltage up to $13 \mathrm{~V}$ compared to $8 \mathrm{~V}$ of pristine network [40], and $4.5 \mathrm{~nm}$ of $\mathrm{ZnO}$ was reported to improve the lifetime of AgNW network from 9.5 to $12 \mathrm{~h}$ at $125 \mathrm{~mA} \mathrm{~cm}^{-2}$ [10]. The imported metal oxide is purer and denser than the previous carbon solution, but the similar deposition process and brittle nature with traditional ITO transparent electrode make it less advantage to low cost and flexible optoelectronics. Whatever the strategy is, so far the reported best lifetime of AgNW composite only extends to hours at high current density and less than half a month at low current flow (Table 1), which is far away from the requirement of long-term operating devices. Furthermore, we still hardly know the failure mechanism of AgNW composite, and lack a good sample with long-term electrical stability to enable the failure mechanism study. 
Here we identified a highly electrical stable AgNW composite with biopolymer whose conductivity remained good after more than $24000 \mathrm{~h}$ under high current density of $100 \mathrm{~mA} \mathrm{~cm}^{-1}$. We thoroughly investigated the failure mechanism of the AgNW composite transparent electrode under different conditions via various characterizations. Wave-break perpendicular to current flow direction is found to be the main failure in AgNW composite electrode instead of local partial failure or uniform degradation across the film. The patterned AgNW composite in microscale exhibits similar long lifetime in resisting current stress as the bulk composite film. The effect of electrical stress, environment surrounding the electrode, chemical corrosion, and over-coating were studied.

\section{Experimental}

\section{Materials}

AgNW in iso-propanol (IPA) dispersion was purchased from Zhejiang Kechuang Advanced Materials Company, with $\sim 30 \mathrm{~nm}$ in diameter and $\sim 15 \mu \mathrm{m}$ in length. Chitosan was obtained from Energy Chemical with deacetylation of $94.6 \%$ and $100-200$ mPas. PEDOT:PSS was provided by Cool Chemistry Technology (Beijing) Co., Ltd. Polydimethylsiloxane (PDMS) was prepared by mixing the Sylgard 184 elastomer base and curing agent (Dow Corning) at the weight ratio of $10: 1$.

\section{Fabrication of Chi-AsA/AgNW composite film}

The scheme of the fabrication process is presented in Fig. S1. Chi-AsA solution with chitosan concentration of $1 \mathrm{wt} \%$ was prepared as reported previously [14]. Diluted the AgNW dispersion with IPA to $0.2 \mathrm{wt} \%$ and shook it to disperse AgNWs uniformly. Firstly coated the AgNW solution on clean glass or PI substrates by rod coating method. AgNW films with different deposition densities were prepared by controlling the number of coating times. Then the prepared Chi-AsA solution was spin-coated on the AgNW film at $3000 \mathrm{rpm}$ for $60 \mathrm{~s}$, followed with drying at $110^{\circ} \mathrm{C}$ for $10 \mathrm{~min}$. The thickness of the Chi-AsA layer on the network void was measured to be about $55 \mathrm{~nm}$ as reported previously [14], and the Chi-AsA on the top of AgNWs is expected to be much thinner than that. The Chi-AsA/AgNW composite film or pristine AgNW film was swabbed into rectangle with $0.8 \mathrm{~cm}$ width by wet cotton swab. Finally, silver paste was cured at the two ends of the samples with $0.8 \mathrm{~cm}$ separation distance, thus the effective area was $0.8 \mathrm{~cm} \times 0.8 \mathrm{~cm}$.

\section{Characterization of the samples}

Scanning electron microscopy (SEM) images were obtained using Zeiss ultra 55 field-emission scanning electron microscope with SE2 detector. Transmittance was measured over the wavelength range of 300$900 \mathrm{~nm}$ on a T6 New Century UV spectrophotometer, and the data at $550 \mathrm{~nm}$ is used to describe the transmittance of the film. X-ray photoelectron spectroscopy (XPS) was obtained with an Axis Ultra DLD electron spectrometer from Kratos using Al target. The thermal images were recorded by FLIR T640 IR thermometer. 


\section{Stability Test}

As shown in Fig. 1, the samples were connected in series in the circuit. When a sample failed, it was removed and the circuit was reconnected. The constant current was applied using a Tektronix PWS4602 current generator, and the data of voltage, current and temperature were collected and recorded by an Agilent 34970A acquisition unit. The resistance was calculated from the ratio of voltage to current. Resistances of the failed samples were measured with a VICTOR VC9808 digital multimeter, which showed that all the resistances were beyond $2000 \Omega$. It is noted that the current density of $100 \mathrm{~mA} \mathrm{~cm}^{-1}$ in this work means that $80 \mathrm{~mA}$ current passes through the film with $0.8 \mathrm{~cm}$ width. The current density of $100 \mathrm{~mA} \mathrm{~cm}^{-1}$ is equivalent to $125 \mathrm{~mA} \mathrm{~cm}^{-2}$ if the current density is defined as dividing the current by the effective area, so as $200 \mathrm{~mA} \mathrm{~cm}^{-1}$ equivalent to $250 \mathrm{~mA} \mathrm{~cm}^{-2}$. Both methods have been used in the literature to define current density $[9-10,29]$.

\section{Results And Discussion}

As shown in Fig. 1a, we studied the electrical stability of pristine AgNW network film and chitosanascorbic acid (Chi-AsA)/AgNW composite film. The IR thermal images show the broken pristine AgNW film while the composite film is still in good state. To test multiple samples simultaneously, we built up an electrical measurement system as shown in Fig. 1b. The samples were connected in series through a screw type wire connector with current resource, and in parallel with voltage measuring system. In this way, we can keep the same current flow into every sample, and measure the individual voltage from every sample. We fixed the initial resistance of the films around $13 \Omega$. Constant current mode was applied to maintain the current density at $100 \mathrm{~mA} \mathrm{~cm}^{-1}$ and $200 \mathrm{~mA} \mathrm{~cm}^{-1}$, respectively. The lifetime is defined until the moment when the sample lost conductivity under current stress $[9,29]$. As shown in Fig. 1c, the pristine AgNW film demonstrated almost linearly increase in resistance from 13 to $32 \Omega$ under $100 \mathrm{~mA}$ $\mathrm{cm}^{-1}$ and then spiked up at $729 \mathrm{~h}$, suggesting the disconnection among AgNW networks at this point. In contrast, Chi-AsA/AgNW composite film maintained stable conductivity for more than $24000 \mathrm{~h}$, and its resistance only slightly changed from 13 to $18 \Omega$. The electrical stability is significantly improved after introduction of Chi-AsA to AgNW networks. We further increased the current density to $200 \mathrm{~mA} \mathrm{~cm}^{-1}$. The composite film showed resistance change below $30 \%$ during a long duration of $846 \mathrm{~h}$, and maintained in good condition until $889 \mathrm{~h}$, while the pristine one quickly exceeded $30 \%$ in resistance only after $34.5 \mathrm{~h}$ and completely lost its conductivity in $82 \mathrm{~h}$ (Fig. 1d). Although lifetime drops predictably with current density, the lifetime of composite at $200 \mathrm{~mA} \mathrm{~cm}^{-1}$ is still longer than that of the pristine film at $100 \mathrm{~mA} \mathrm{~cm}{ }^{-1}$. Since patterning technology is essential for electronic devices, we further tested the patterned films in a width of $350 \mu \mathrm{m}$ at the current density of $200 \mathrm{~mA} \mathrm{~cm}^{-1}$. It is found the pattern has similar long lifetime in resisting current stress as the bulk composite film as shown in Fig. 1e. As illustrated in Fig. $1 \mathrm{f}$ and Table 1, the previously reported composite AgNW films mostly operated less than hundreds of hours at the current density below $100 \mathrm{~mA} \mathrm{~cm}{ }^{-2}$. Our composite worked normally for more than $24000 \mathrm{~h}$ under $100 \mathrm{~mA}$ $\mathrm{cm}^{-1}$ (i.e. $125 \mathrm{~mA} \mathrm{~cm}^{-2}$ ), exhibiting much better stability under current loading. As most devices operate 
below $100 \mathrm{~mA} \mathrm{~cm}^{-1}$, the composite film with improved electrical stability to $\sim 3$ years at $100 \mathrm{~mA} \mathrm{~cm}^{-1}$ could enable long-term stable devices to meet the commercial longevity requirement.

The mechanism behind the improvement of electrical stability is highly interesting. First, we used dualprobe ohmmeter to check the conductivity across the failed film after longevity current loading test. As shown in Fig. 2a, one probe of the ohmmeter was secured at silver paste connection electrode coated on one side of the composite film, and another probe was moved freely to contact with any points. Afterwards, change the fixed probe to another silver paste contact electrode and repeat the check. We found that actually most regions across the failed film still had good conductivities, and the resistances only slightly shifted to $\sim 20-40 \Omega$. However, there was always one site with resistance larger than $2000 \Omega$ when the probe was moved up and down, perpendicular to the current flow direction. It suggests a disconnection line perpendicular to the current flow. We then used IR thermal camera to track the failure process, and found most samples kept uniform thermal distribution in the early stage, and then local hot spots showed up and grew to a wave line in the latter stage (Fig. 2b). It demonstrates that the network undergoes slow and widespread degradation for a period. After that, the AgNWs in some weak regions degrade to a critical state and begin accelerated degradation, appearing as hot spots. Then the current in the broken area redistributes to the surroundings vertical to current flow. The increased current density and corresponding higher temperature accelerate the degradation of surroundings, and in turn, affect further region. Thus, the crack extends vertically to the current flow in this cascade process. When the remaining AgNWs along the wave break are insufficient to withstand the crowded current, rapid electrical failure occurs in the AgNW film, consistent with the phenomenon in Fig. 1c-d.

The optical and SEM images further confirmed the failure mode. As shown in Fig. 2(c, f, i), an obvious waved break formed in Chi-AsA/AgNW composite network and pristine AgNW network. In the enlarged SEM images, the composite electrode shows smooth silver nanowire without obvious damage in the region aside the waved break (Fig. 2d), and serious silver migration inside the break (Fig. 2e). It suggests that the degradation is only limited along the wave break. Such distinct degradation in different positions has been seldom reported in long-term electrical failure of AgNW composite electrode. In the enlarged SEM images of pristine AgNWs, serious damages are observed inside the waved crack, and across the whole film as well. Disconnections in the cross-linked junctions and hillocks on surface of nanowires appeared both at 200 and $100 \mathrm{~mA} \mathrm{~cm}^{-1}$ (Fig. 2g-k). So compared to the composite, two main differences are found. First, inside the waved crack, the silver migration was mainly limited along the nanowire and less nanoparticles formed among network voids in AgNW composite, while the silver migrated more freely in pristine one. Nanowires fractured into particles, migrated into aggregations, and moved to network voids. Second, in the region aside the waved crack, silver nanowire kept its original shape and morphology well in composite AgNWs, while degradation appeared everywhere in pristine one. We could hypothesize that the over-coating layer efficiently stopped the silver migration under current. Besides, several local failures similar with the reported hotspots were also found in the AgNW composites (Fig. S2), especially when there is an external contaminant nanoparticle on the surface. 
The position of wave break in pristine samples depends on the role of Joule heating and electromigration in the failure mechanism as we reported [44]. The wave break locates in the middle region of the film when rapid thermal fusing dominates the short-term failure, and moves towards anode when electromigration and electrochemical effects play a role in the long-term failure [44]. We sum up the locations of the wave break of failed samples with initial resistance of $10 \sim 15 \Omega$ at $200 \mathrm{~mA} \mathrm{~cm}^{-1}$ in Fig. S3. For pristine samples, the wave break mainly occurs near the anode. By contrast, the composite samples show a more complicated failure locations, which is regarded as the result of the effective inhibition on electromigration by Chi-AsA coating. Due to the suppression of electromigration near the electrode in composite samples, the influence of other factors, such as high temperature in the center area, local inhomogeneity of the network, and impurities in the film, might be prominent, resulting in various break locations.

We then studied the function of Chi-AsA coating via changing the covering position shown in Fig. 3a. First, the Chi-AsA was only coated on the central area of AgNW networks and exposed the area near connection electrodes (Sample I). The lifetime at current density of $200 \mathrm{~mA} \mathrm{~cm}^{-1}$ was drastically reduced from 889 to $116 \mathrm{~h}$ compared with fully-covered composite film (Fig. 3b). The wave break appeared at the exposed AgNWs area next to covered area in the side towards positive connection electrode, exhibiting obvious migration of silver to form spheres (Fig. 3c). Second, the Chi-AsA coating was extended to the whole area of AgNW networks, but the part beneath connection electrodes was still the bare AgNWs (Sample II). The sample exhibited improved lifetime to $561 \mathrm{~h}$, and the wave break located at the vicinity of the positive connection electrode (Fig. 3e). It is similar with Sample I, in which the wave break both happened near the boundary of Chi-AsA coating. Third, only around $30 \%$ of the AgNW network in the central area was exposed, and all other area including the part beneath connection electrodes was coated with Chi-AsA (Sample III). It showed a lifetime of $382 \mathrm{~h}$, which is much higher than the first sample type, but lower than the second one. The wave break line appeared at the border between the covered and the exposed networks with serious silver migration (Fig. 3g). Pristine AgNWs inside exposed area also exhibited obvious degradation with disconnected nanoparticles on the surface of AgNWs and gaps among network, and some big cluster of silver agglomeration at the contact junctions (Fig. 3h). For all three types of samples, AgNWs covered with Chi-AsA remained intact network, good contact junctions and smooth surface after the electrical failure of the samples (Fig. 3d, f, i).

It demonstrates the effectiveness of Chi-AsA protection on electrical stability. The main failure always occurred at the boundary region of Chi-AsA coating no matter where it is. When the location of Chi-AsA covering layer moves, the wave break line follows instead of only appearing near the connection electrode. Inside the failure area of wave break, rut voids along the deposition path of silver nanowire left after silver migration, which is similar with the morphology of fully-covered composite samples. It is considered that the silver atoms must break through the obstacles of Chi-AsA to migrate. Since the ChiAsA polymer is coated on the surface and fills the voids of AgNW network, silver atoms are strongly suppressed from moving along the AgNWs or migrating to the surrounding area under current stress. Thus, the electrical and thermal stability of Chi-AsA/AgNW film is significantly enhanced. In addition to 
electromigration, the degradation caused by concentrated Joule heating at the junctions also contributes to the electrical failure $[22,36]$. Since Chi-AsA layer helps form a tighter contact and smaller contact resistance between AgNWs [14], the risk of contact junctions being molten with Joule heating is reduced compared with pristine AgNW networks. Besides, it is learned from sample II that even all AgNWs are covered with Chi-AsA, the lifetime under current stress is still lower than that of the fully covered one in which the part beneath connection electrode is also protected with Chi-AsA. It indicates that the protection of AgNWs beneath conductive connection points is also important. It requires the coating material be thin enough to transfer electron transfer between AgNW networks and connection electrodes, but strong enough to provide sufficient protection on the AgNWs from current stress. Some traditional insulating polymers such as polydimethylsiloxane or epoxy can also protect AgNW networks as overcoatings, but generally make the composite surface insulate from the top connection electrode. In contrast, Chi-AsA layer with tens of nanometers can deposit on AgNWs beneath connection points with unchanged conductivity and good protection.

According to electrical failure theory of bulk metal film, electromigration highly depends on the current density and temperature. The current density on single AgNW is related to both applied current value and network density. We fabricated pristine and composite samples with various AgNWs deposition densities by different coating times. The higher deposition density represents lower transmittance. As shown in Fig. S4, the transmittance at $550 \mathrm{~nm}$ decreases linearly by $1.1 \%$ on average for each additional coating time. We investigated the electrical stability of the composite films with 2 7 coating times and corresponding transmittance of 96.8 91.6\%. As shown in Fig. 4a-b, the lifetime of the composite samples increases obviously with AgNWs deposition density. For example, composite film with $96.1 \%$ of transmittance only subsisted for $223 \mathrm{~h}$ at current density of $200 \mathrm{~mA} \mathrm{~cm}^{-1}$, which expanded to $1582 \mathrm{~h}$ for the one with $92.5 \%$ of transmittance. Pristine samples also exhibit the similar relationship between lifetime and transmittance, but the lifetime is below $10 \%$ of the composite sample with comparable deposition density. Higher deposition density means lower current density on single AgNW, resulting in slighter electromigration, less Joule heating, and hence better electrical stability. In addition, denser AgNW networks benefit more uniform distribution of current, and thus reduce the occurrence of hotspots [21, 23, $29,45]$. The AgNWs deposition density can be characterized with SEM, and the current density along single AgNW is calculated via dividing the current by the sum of cross-sectional area of all AgNWs on the line perpendicular to current flow as shown in Fig. S5. The calculated current density on single AgNW from SEM images is inversely proportional to the coating times (Fig. S6), which verifies the accuracy of the calculation from SEM images. As shown in Fig. 4c, it demonstrates a power function relationship with a power index of -3.7 between lifetime and current density on single AgNW. It illustrates that the lifetime could exceed $1000 \mathrm{~h}$ if the current density of single AgNW is less than $8.4 \times 10^{5} \mathrm{~A} \mathrm{~cm}^{-2}$, which is approximately corresponding to the films with $94 \%$ of transmittance at $200 \mathrm{~mA} \mathrm{~cm}^{-1}$, or $97 \%$ of transmittance at $100 \mathrm{~mA} \mathrm{~cm}^{-1}$. Therefore, as long as the transmittance is among the application requirement, increasing the AgNWs deposition density would significantly improve the lifetime under current stress. 
We then studied the performance of composite and pristine AgNWs under elevated current densities, and compared their tolerance threshold to current shock. The AgNW networks were fabricated on polyimide (PI) in this experiment because it can withstand higher current density than glass before brittle fracture induced by thermal stress. Meanwhile, the network on PI substrate exhibits higher temperature than that on glass under similar current stress because of different heat dissipation and thermal capacity, which results in more resistance change. The pristine and composite films with initial resistance of $14 \Omega$ were tested under current density that started from $50 \mathrm{~mA} \mathrm{~cm}{ }^{-1}$ and increased step by step of $25 \mathrm{~mA} \mathrm{~cm}^{-1}$ per 10 min. As shown in Fig. 4d, the resistance increased with current density because of higher temperature due to Joule heating. When the current density was below $175 \mathrm{~mA} \mathrm{~cm}^{-1}$, the composite and pristine films exhibited similar resistance. However, the composite film started to exhibit obvious smaller resistance than the pristine film at $200 \mathrm{~mA} \mathrm{~cm}^{-1}$, meaning slower aging process in this condition. The pristine film failed at the current density of $250 \mathrm{~mA} \mathrm{~cm}^{-1}$ when the substrate was intact. By contrast, the ChiAsA/AgNW composite film performed well until $350 \mathrm{~mA} \mathrm{~cm}^{-1}$ before the rupture of substrate. It illustrates that the Chi-AsA/AgNW composite film itself can withstand current shock above $350 \mathrm{~mA} \mathrm{~cm}^{-1}$ on a stronger substrate, much superior to pristine AgNW film.

To understand the influence of temperature on the electrical reliability of composite films, we studied the effect of Chi-AsA coating on the heat dissipation of AgNW network. The film temperature increased after current applied due to Joule heating, and reached steady stage in several minutes (Fig. 5a). The temperature increase is defined as the difference between the room temperature $\left(\mathrm{T}_{0}\right)$ and steady temperature. As shown in Fig. 5b, the temperature increase changes linearly with the resistance. Both the composite and pristine samples meet the same linear fit at $100 \mathrm{~mA} \mathrm{~cm}^{-1}$ and $200 \mathrm{~mA} \mathrm{~cm}^{-1}$, respectively. It suggests that the Chi-AsA coating shows no adverse effect on the heat dissipation of AgNW network. Tens of nanometers thickness of the coating layer is negligible relative to the substrate and shows minimal obstacle to surface heat dissipation. Besides, Chi-AsA enables stronger adhesion between AgNWs and substrate [14], which enhances the heat dissipation through substrate.

Both the internal Joule heating and external thermal environment contribute to the temperature of AgNW films, and thus influence their electrical stability. Chi-AsA/AgNW composite samples of $13 \Omega$ were applied with current from a DC power and heated on a hot plate simultaneously. The sample temperature was controlled by the current density and/or hot plate temperature. Compared with the composite film at 200 $\mathrm{mA} \mathrm{cm}^{-1}$ without external heating ( $50^{\circ} \mathrm{C}$ due to internal Joule heating), the lifetime of composite film was shortened from $889 \mathrm{~h}$ to $450 \mathrm{~h}$ at $200 \mathrm{~mA} \mathrm{~cm}{ }^{-1} / 60^{\circ} \mathrm{C}$, and further to $136 \mathrm{~h}$ at $200 \mathrm{~mA} \mathrm{~cm}^{-1} / 85^{\circ} \mathrm{C}$ (Fig. 5c). Most area except the wave break of the network at $200 \mathrm{~mA} \mathrm{~cm}^{-1} / 85^{\circ} \mathrm{C}$ showed degradation with breakage and aggregations (Fig. 5c- $\varangle$ ), and that at $200 \mathrm{~mA} \mathrm{~cm}-1 / 60{ }^{\circ} \mathrm{C}$ showed some fractures (Fig. $5 c-\varangle)$. It is expected that high temperature not only enhances thermal melting, but also provides energy for silver atoms to migrate and accelerates the corrosion, causing poor electrical stability of the film.

Interestingly, although temperature shows significant effect on electrical stability, the lifetime of the sample at same temperature is heavily dependent on current stress. As shown in Fig. 5c, the composite 
film at $100 \mathrm{~mA} \mathrm{~cm}-1 / 85^{\circ} \mathrm{C}$ showed a lifetime of $866 \mathrm{~h}$, about 6.4 times that at $200 \mathrm{~mA} \mathrm{~cm}-1 / 85^{\circ} \mathrm{C}$, and only slight damages were observed in most area (Fig. $5 \mathrm{c}-\mathrm{Z}$ ). Meanwhile, the composite film at $85^{\circ} \mathrm{C}$ without current stress only increased $5 \Omega$ in resistance after more than $2000 \mathrm{~h}$, which maintained intact network without obvious fragmentation (Fig. $5 \mathrm{c}-\mathrm{\Xi}$ ). It means that even at the same macroscopic temperature, the electrical stability still depends on the applied current density. The effect of current stress is not only to generate Joule heating, but also causing electromigration due to momentum exchange and electrochemical action. In addition, as the source of Joule heating, AgNW network only accounts for a small percentage of the entire film surface area. Thus, the temperature of individual AgNW, particularly at the contact junctions, is expected to be higher than the apparent macroscopic temperature. Even at the same macroscopic temperature by current stress and external heat simultaneously, higher current density actually results in more Joule heating on individual AgNW and thus reduced lifetime.

The importance of the current density to the electrical stability is further confirmed by the comparison of different samples at same temperature. The composite films of $28.8 \Omega / 100 \mathrm{~mA} \mathrm{~cm}^{-1}$ and $8.5 \Omega / 200 \mathrm{~mA}$ $\mathrm{cm}^{-1}$ both reached the apparent temperature of $43^{\circ} \mathrm{C}$. However, the $8.5 \Omega / 200 \mathrm{~mA} \mathrm{~cm}^{-1}$ film showed a lifetime much longer than that of the $28.8 \Omega / 100 \mathrm{~mA} \mathrm{~cm}^{-1}$ film as shown in Fig. $5 \mathrm{~d}$. To achieve the same macroscopic temperature, individual AgNW in sparser network (28.8 $\Omega$ ) should produce more Joule heat, which means higher current density in single AgNW and thus shorter lifetime. Therefore, the current density and temperature in single AgNW are the more important parameters in evaluating the long-term electrical reliability of the network than the apparent macroscopic temperature of the film.

In addition to electromigration and Joule heating, the effect of corrosion on the electrical stability cannot be ignored as well. Ambient substances such as sulfides, oxygen and moisture have been reported to cause corrosion of AgNWs, and sulfidation has been proven to be the dominant effect [24-26]. The corrosion is expected to function synergistically with electromigration and Joule heating, and even probably brings about complex electrochemical effect, thereby accelerating the electrical failure. XPS was taken from the failed pristine and composite films in Fig. 1c-d. As shown in Fig. 6, the ratio of silver to sulfur content was 82: 18 in the pristine AgNW film that sustained for $729 \mathrm{~h}$ under $100 \mathrm{~mA} \mathrm{~cm}^{-1}$. The ratio drops to 96: 4 for the pristine AgNW film under $200 \mathrm{~mA} \mathrm{~cm}^{-1}$ for $82 \mathrm{~h}$. It suggests that corrosion by sulfur took place under current stress and developed over time. The corrosion of silver decreases the nanowire diameter and thus degrades the network conductivity, causing elevated temperature and enhanced current density in AgNWs, which plays a role in long-term failure process under current. Coating is an effective way to inhibit the corrosion. Remarkably, even though the Chi-AsA/AgNW composite film underwent longer time and higher temperature test at $200 \mathrm{~mA} \mathrm{~cm}^{-1}$ than the pristine film at $100 \mathrm{~mA} \mathrm{~cm}^{-1}$, XPS showed no obvious signal of sulfur, demonstrating good resistance to corrosion by ambient chemicals.

AgNW networks are usually integrated with other materials in optoelectronic devices. Some materials are harmful to the stability of AgNW network. AgNW film is often compounded with PEDOT:PSS when used in OLEDs and solar cells as electrode. We imported PEDOT:PSS onto the top of AgNW film and Chi- 
AsA/AgNW film, both with initial resistance of $10 \Omega$, and then tested the electrical stability at $200 \mathrm{~mA} \mathrm{~cm}^{-}$ ${ }^{1}$. As shown in Fig. 7, the lifetime of PEDOT:PSS/AgNW film decreased to merely $109 \mathrm{~h}$ compared to 156 $\mathrm{h}$ of pristine AgNW film. The result is consistent with the previously reported work that the acidic property and water-absorbing tendency of commercial PEDOT:PSS lead to declining stability of AgNW network [41]. By contrast, the PEDOT:PSS/Chi-AsA/AgNW film subsisted for $751 \mathrm{~h}$. The results illustrate that the anti-permeation property of Chi-AsA coating not only benefits resisting ambient corrosion, but also contributes to suppressing the destructive effect of composite materials on AgNW network. AgNW network is often packaged for protection such as in film heaters. Here we used PDMS as a protective cover on the top of the sample. The PDMS/AgNW sample exhibited improved lifetime of $792 \mathrm{~h}$ at $200 \mathrm{~mA}$ $\mathrm{cm}^{-1}$. Meanwhile, the lifetime was significantly elevated to $4193 \mathrm{~h}$ for the PDMS/Chi-AsA/AgNW composite film. It demonstrates that no matter what kind of materials are composited with, the ChiAsA/AgNW composite films always show superior advantages in electrical stability in contrast to pristine AgNW films.

\section{Conclusion}

Chi-AsA/AgNW composite transparent conductive film exhibited excellent long-term electrical stability under current stress. With initial resistance of $13 \Omega$, the Chi-AsA/AgNW composite film operated functionally at the current density of $100 \mathrm{~mA} \mathrm{~cm}^{-1}$ for more than $24000 \mathrm{~h}$ without significant damage, while the pristine AgNW film failed within $729 \mathrm{~h}$. The electrical failure mode demonstrates the wave break perpendicular to the current flow direction in the composite film rather than uniform degradation of the entire network. Chi-AsA can prevent Ag atoms from migration, reduce the Joule heating at the contact junctions, and inhibit the corrosion of AgNWs, resulting in robust AgNW networks resisting current shock. The electrical stability is highly related to the temperature and current density along individual silver nanowire, and the lifetime increases remarkably with lower environment temperature and better film conductivity. Even in harsher environment or with sparser AgNW densities, the Chi-AsA/AgNW composite film exhibites advantages in electrical stability compared to the pristine AgNW network, which demonstrates a good potential in practical application.

\section{Declarations}

Conflicts of interest

There are no conflicts to declare.

Acknowledgements

This study was sponsored by National Natural Science Foundation of China under Grant 51603043.

\section{References}


1. Ye, A. R. Rathmell, Z. Chen, I. E. Stewart, B. J. Wiley, Metal nanowire networks: the next generation of transparent conductors. Adv. Mater. 26, 6670-6687 (2014)

2. Ye, J. Yan, S. Xie, Y. Kong, T. Liang, H. Chen, M. Xu, Silver nanowire-graphene hybrid transparent conductive electrodes for highly efficient inverted organic solar cells. Nanotechnology 28, 305402 (2017)

3. H. Seo, S. Lee, K. H. Min, S. Chandramohan, A. H. Park, G. H. Lee, M. Park, E. K. Suh, M. J. Kim, The role of graphene formed on silver nanowire transparent conductive electrode in ultra-violet light emitting diodes. Sci. Rep. 6, 29464 (2016)

4. Gupta, K. D. Rao, S. Kiruthika, G. U. Kulkarni, Visibly transparent heaters. ACS Appl. Mater. Interfaces 8, 12559-12575 (2016)

5. Lee, P. Lee, H. Lee, D. Lee, S. S. Lee, S. H. Ko, Very long Ag nanowire synthesis and its application in a highly transparent, conductive and flexible metal electrode touch panel. Nanoscale 4, 6408-6414 (2012)

6. Yao, Y. Zhu, Wearable multifunctional sensors using printed stretchable conductors made of silver nanowires. Nanoscale 6, 2345-2352 (2014)

7. Li, X. Liu, X. Chen, W. Y. Lai, W. Huang, A simple strategy towards highly conductive silver-nanowire inks for screen-printed flexible transparent conductive films and wearable energy-storage devices. Adv. Mater. Technol. 4, 1900196 (2019)

8. Liang, T. Zhao, W. Jiang, X. Yu, Y. Hu, P. Zhu, H. Zheng, R. Sun, C.-P. Wong, Highly transparent triboelectric nanogenerator utilizing in-situ chemically welded silver nanowire network as electrode for mechanical energy harvesting and body motion monitoring. Nano Energy 59, 508-516 (2019)

9. H. Khaligh, I. A. Goldthorpe, Failure of silver nanowire transparent electrodes under current flow. Nanoscale Res. Lett. 8, 235 (2013)

10. Chen, F. Zhao, K. Tong, G. Saldanha, C. Liu, Q. Pei, Mitigation of electrical failure of silver nanowires under current flow and the application for long lifetime organic light-emitting diodes. Adv. Electron. Mater. 2, 1600167 (2016)

11. Zhang, X. Yan, J. Chen, J. Zhao, Large-size graphene microsheets as a protective layer for transparent conductive silver nanowire film heaters. Carbon 69, 437-643 (2014)

12. Kwon, K. Kim, J. Heo, I. Yi, I. Chung, Study on Ag mesh/conductive oxide hybrid transparent electrode for film heaters. Nanotechnology 25, 265702 (2014)

13. Ji, W. He, K. Wang, Y. Ran, C. Ye, Thermal response of transparent silver nanowire/PEDOT:PSS film heaters. Small 10, 4951-4960 (2014)

14. Jin, Y. Sun, K. Wang, Y. Chen, Z. Liang, Y. Xu, F. Xiao, Long-term stable silver nanowire transparent composite as bottom electrode for perovskite solar cells. Nano Res. 11, 1998-2011 (2018)

15. Ahn, Y. Jeong, Y. Lee, Improved thermal oxidation stability of solution-processable silver nanowire transparent electrode by reduced graphene oxide. ACS Appl. Mater. Interfaces 4, 6410-6414 (2012) 
16. Lee, H. Lee, Y. Ahn, Y. Jeong, D. Y. Lee, Y. Lee, Highly stable and flexible silver nanowire-graphene hybrid transparent conducting electrodes for emerging optoelectronic devices. Nanoscale 5, 77507755 (2013)

17. T. Camic, F. Oytun, M. H. Aslan, H. J. Shin, H. Choi, F. Basarir, Fabrication of a transparent conducting electrode based on graphene/silver nanowires via layer-by-layer method for organic photovoltaic devices. J Colloid Interface Sci. 505, 79-86 (2017)

18. Zhang, J. Wu, H. Liu, J. Wang, X. Zhao, Z. Xie, Efficient flexible polymer solar cells based on solutionprocessed reduced graphene oxide-assisted silver nanowire transparent electrode. Org Electron. 50, 255-263 (2017)

19. Liang, L. Li, K. Tong, Z. Ren, W. Hu, X. Niu, Y. Chen, Q. Pei, Silver nanowire percolation network soldered with graphene oxide at room temperature and its application for fully stretchable polymer light-emitting diodes. Small 8, 1590-1600 (2014)

20. Dong, Z. Wu, Y. Jiang, W. Liu, X. Li, B. Jiao, W. Abbas, X. Hou, A flexible and thin graphene/silver nanowires/polymer hybrid transparent electrode for optoelectronic devices. ACS Appl. Mater. Interfaces 8, 31212-31221 (2016)

21. Sannicolo, N. Charvin, L. Flandin, S. Kraus, D. T. Papanastasiou, C. Celle, J. P. Simonato, D. MuñozRojas, C. Jiménez, D. Bellet, Electrical mapping of silver nanowire networks: a versatile tool for imaging network homogeneity and degradation dynamics during failure. ACS Nano 12, 4648-4659 (2018)

22. Fantanas, A. Brunton, S. J. Henley, R. A. Dorey, Investigation of the mechanism for current induced network failure for spray deposited silver nanowires. Nanotechnology 29, 465705 (2018)

23. Kumar, N. S. Vidhyadhiraja, G. U. Kulkarni, Current distribution in conducting nanowire networks. J. Appl. Phys. 122, 045101 (2017)

24. Deignanab, I. A. Goldthorpe, The dependence of silver nanowire stability on network composition and processing parameters. RSC Adv. 7, 35590-35597 (2017)

25. L. Elechiguerra, L. Larios-Lopez, C. Liu, D. Garcia-Gutierrez, A. Camacho-Bragado, M. J. Yacaman, Corrosion at the nanoscale: the case of silver nanowires and nanoparticles. Chem. Mater. 17, 6042$6052(2005)$

26. T. Liu, S. X. Huang, Transparent conductive silver nanowire electrodes with high resistance to oxidation and thermal shock. RSC Adv. 4, 59226-59232 (2014)

27. K. Moon, J. I. Kim, H. Lee, K. Hur, W. C. Kim, H. Lee, 2D graphene oxide nanosheets as an adhesive over-coating layer for flexible transparent conductive electrodes. Sci. Rep. 3, 1112 (2013)

28. Chen, M. Li, X. Wen, Y. Yang, D. He, W. C. H. Choy, H. Lu, Enhanced silver nanowire composite window electrode protected by large size graphene oxide sheets for perovskite solar cells. Nanomaterials $\mathbf{9}$, 193 (2019)

29. H. Khaligh, L. Xu, A. Khosropour, A. Madeira, M. Romano, C. Pradére, M. Tréguer-Delapierre, L. Servant, M. A. Pope, I. A. Goldthorpe, The Joule heating problem in silver nanowire transparent electrodes. Nanotechnology 28, 425703 (2017) 
30. S. Alshammari, Improved electrical stability of silver NWs based hybrid transparent electrode interconnected with polymer functionalized CNTs. Mater. Res. Bull. 111, 245-250 (2019)

31. C. Goak, T. Y. Kim, D. U. Kim, K. S. Chang, C. S. Lee, N. Lee, Stable heating performance of carbon nanotube/silver nanowire transparent heaters. Appl. Surf. Sci. 510, 145445 (2020)

32. Cai, X. Piao, X. Yao, W. Gao, E. Nie, Z. Zhang, Z. Sun, Transparent conductive film based on silver nanowires and single-wall carbon nanotubes for transparent heating films. Nanotechnology $\mathbf{3 0}$, 225201 (2019)

33. Chen, L. Song, Z. Tao, X. Shao, Y. Huang, Q. Cui, X. Guo, Neutral-pH PEDOT:PSS as over-coating layer for stable silver nanowire flexible transparent conductive films. Org Electron 15, 3654-3659 (2014)

34. S. Woo, J. T. Han, S. Jung, J. I. Jang, H. Y. Kim, H. J. Jeong, S. Y. Jeong, K. J. Baeg, G. W. Lee, Electrically robust metal nanowire network formation by in-situ interconnection with single-walled carbon nanotubes. Sci. Rep. 4, 4804 (2014)

35. J. Lee, J.-W. Kim, J. H. Park, Y. Porte, J.-H. Kim, J.-W. Park, S. Kim, J.-M. Myoung, SWCNT-Ag nanowire composite for transparent stretchable film heater with enhanced electrical stability. J. Mater. Sci. 53, 12284-12294 (2018)

36. Zhu, T. Wan, P. Guan, Y. Wang, T. Wu, Z. Han, G. Tang, D. Chu, Improving thermal and electrical stability of silver nanowire network electrodes through integrating graphene oxide intermediate layers. J. Colloid Interface Sci. 566, 375-382 (2020)

37. H. Nguyen, J. Resende, D. T. Papanastasiou, N. Fontanals, C. Jiménez, D. Muñoz-Rojas, D. Bellet, Lowcost fabrication of flexible transparent electrodes based on Al doped $\mathrm{ZnO}$ and silver nanowire nanocomposites: impact of the network density. Nanoscale 11, 12097-12107 (2019)

38. Yu, N. Jin, Z. Wang, J. Lin, J. Wei, Q. Luo, C. Q. Ma, Use of solution-processed zinc oxide to prevent the breakdown in silver nanowire networks. Nanotechnology 31, 18 LT01 (2020)

39. Khan, V. H. Nguyen, D. Muñoz-Rojas, S. Aghazadehchors, C. Jiménez, N. D. Nguyen, D. Bellet, Stability enhancement of silver nanowire networks with conformal $\mathrm{ZnO}$ coatings deposited by atmospheric pressure spatial atomic layer deposition. ACS Appl. Mater. Interfaces 10, 19208-19217 (2018)

40. Lagrange, T. Sannicolo, D. Muñoz-Rojas, B. G. Lohan, A. Khan, M. Anikin, C. Jiménez, F. Bruckert, Y. Bréchet, D. Bellet, Understanding the mechanisms leading to failure in metallic nanowire-based transparent heaters, and solution for stability enhancement. Nanotechnology 28, 055709 (2017)

41. Mayousse, C. Celle, A. Fraczkiewicz, J. P. Simonato, Stability of silver nanowire based electrodes under environmental and electrical stresses. Nanoscale 7, 2107-2115 (2015)

42. Lu, D. Zhang, J. Cheng, J. Liu, J. Mao, W. C. H. Choy, Locally welded silver nano-network transparent electrodes with high operational stability by a simple alcohol-based chemical approach. Adv. Funct. Mater. 25, 4211-4218 (2015)

43. J. Choi, S. J. Kim, J. S. Jang, J. H. Lee, I. D. Kim, Silver nanowire embedded colorless polyimide heater for wearable chemical sensors: improved reversible reaction kinetics of optically reduced graphene oxide. Small 12, 5826-5835 (2016) 
44. Wang, Y. Jin, X. Wang, B. Qian, J. Wang, F. Xiao, Investigation into the failure mechanism of silver nanowire network film under electrical stress. 2020 IEEE 70th Electronic Components and Technology Conference (ECTC). Orlando, FL, USA (2020)

45. W. Cheuk, K. Pei, P. K. L. Chan, Degradation mechanism of a junction-free transparent silver network electrode. RSC Adv. 6, 73769-73775 (2016)

\section{Tables}

Due to technical limitations, table 1 is only available as a download in the Supplemental Files section.

\section{Figures}


(a)

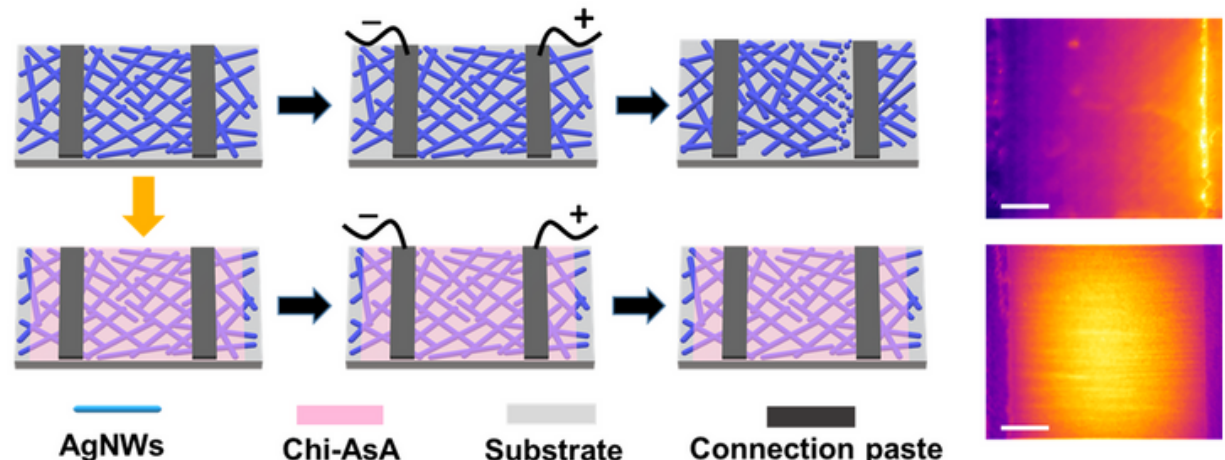

AgNWs Chi-AsA Substrate Connection paste

(b)
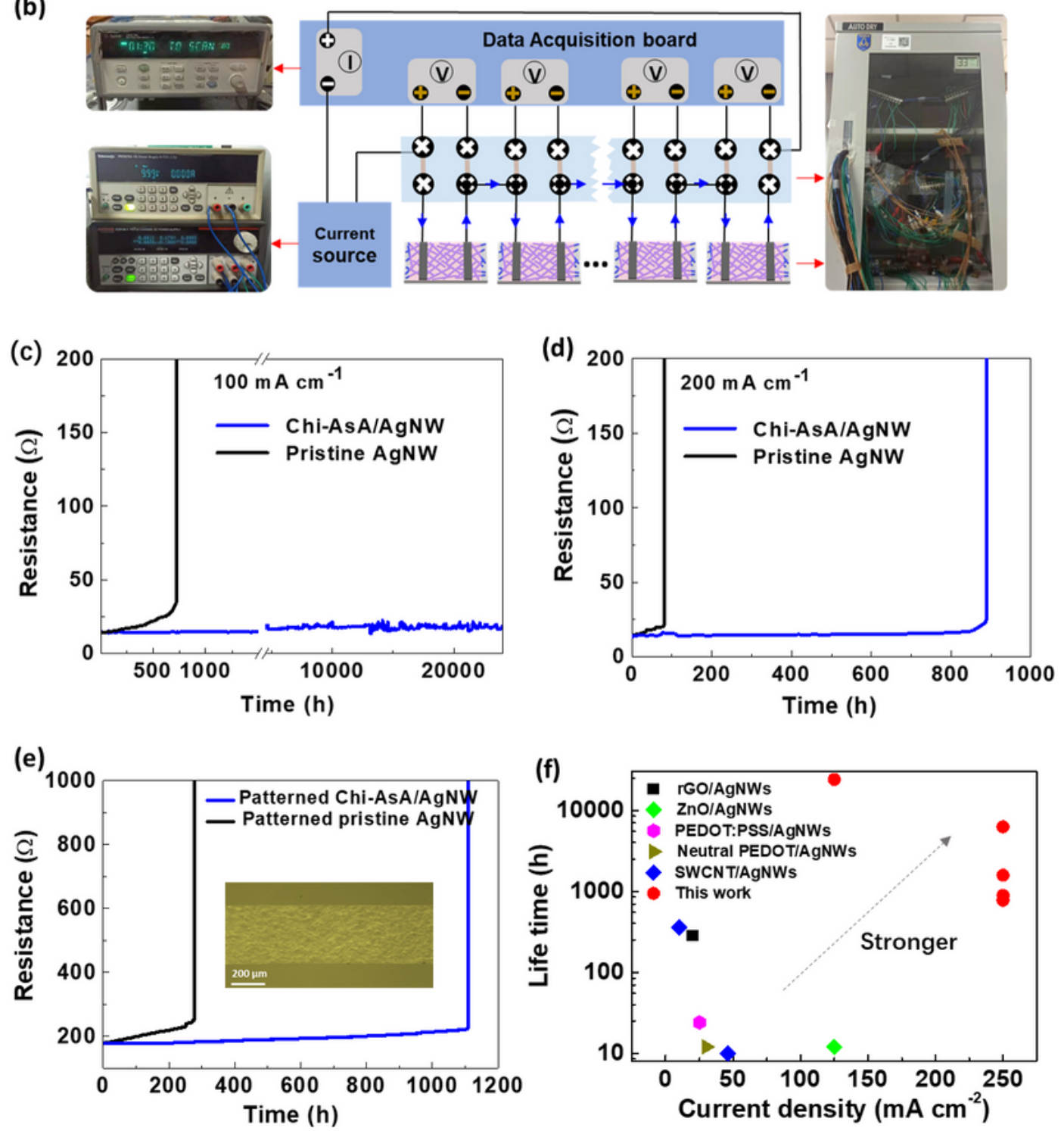

Figure 1

a Schematic structure of the pristine AgNW sample and Chi-AsA/AgNW composite sample, and corresponding IR images after $82 \mathrm{~h}$ at $200 \mathrm{~mA} \mathrm{~cm}-1$. The bar is $2 \mathrm{~mm}$. b Photograph and schematic illustration of the system for long-term electrical stability test. c-d Resistance as a function of time for pristine AgNW film and Chi-AsA/AgNW composite film at $100 \mathrm{~mA} \mathrm{cm-1} \mathrm{(c)} \mathrm{and} 200 \mathrm{~mA} \mathrm{cm-1} \mathrm{(d),}$ respectively. e Resistance as a function of time for patterned pristine AgNW film and patterned Chi- 
AsA/AgNW composite film with a width of $350 \mu \mathrm{m}$ at $200 \mathrm{~mA} \mathrm{~cm}-1$. $\mathrm{f}$ Comparison of the electrical lifetime of AgNW composite films under various current density.

(a)
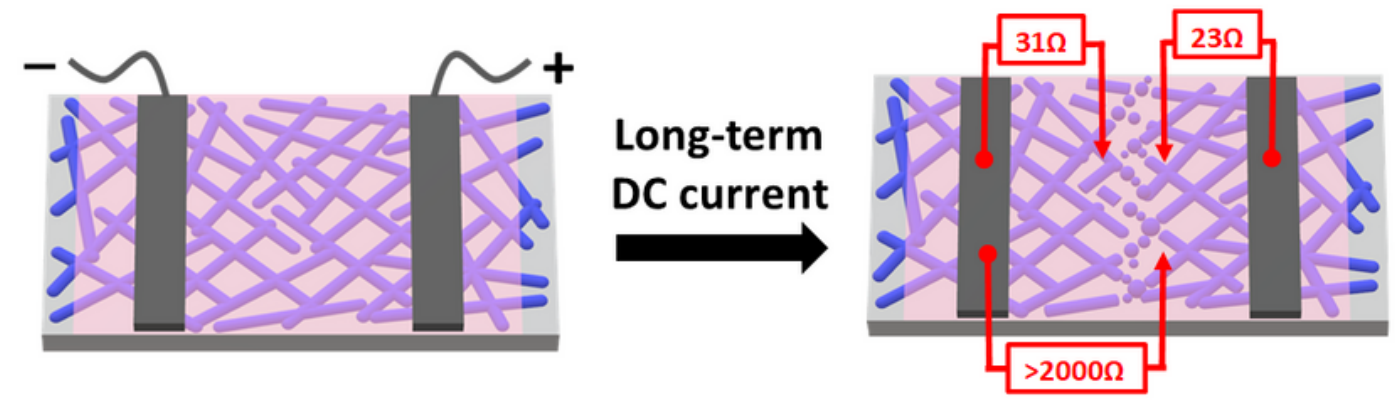

(b)
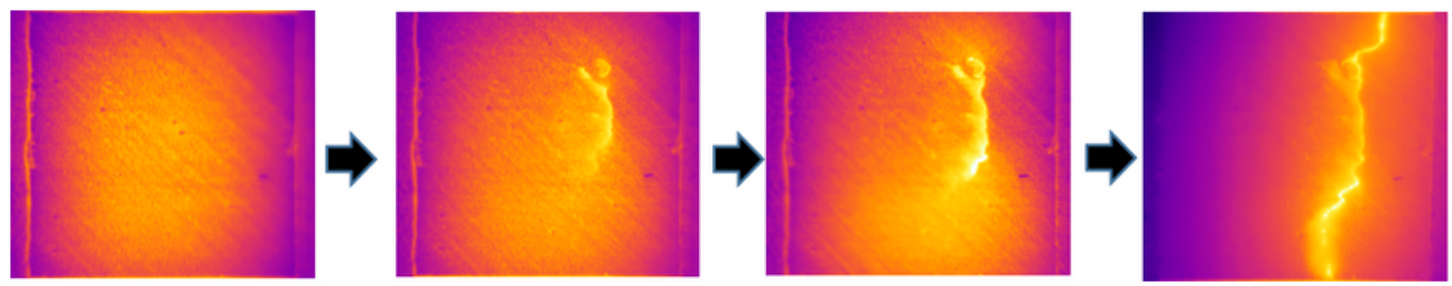

(c)

(d)
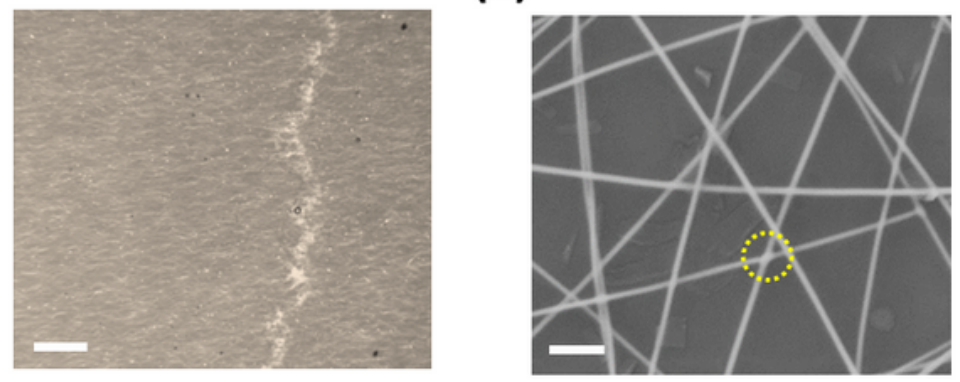

(e)

(f)

(g)
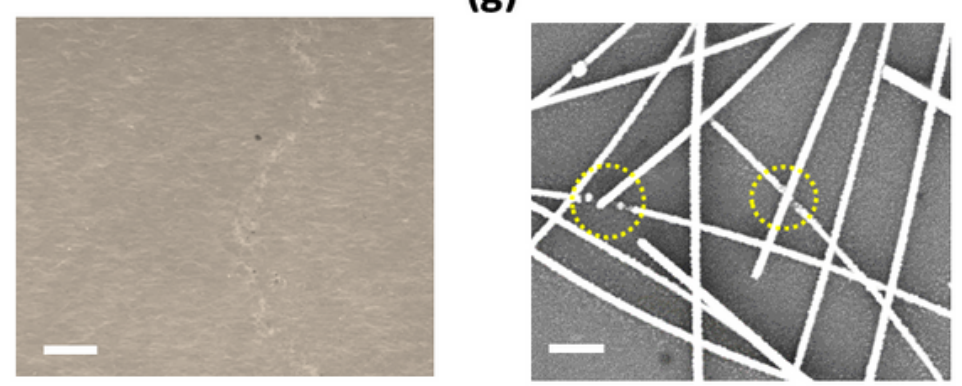

(i)

(j)
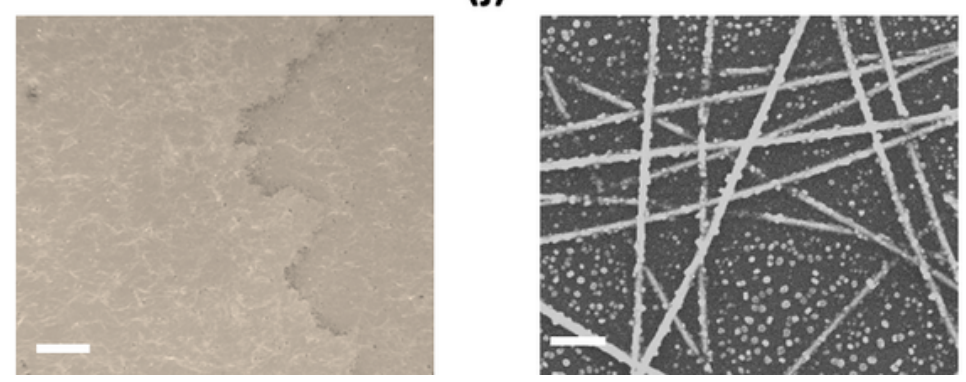

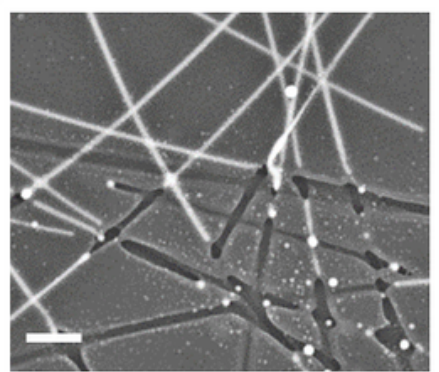

(h)

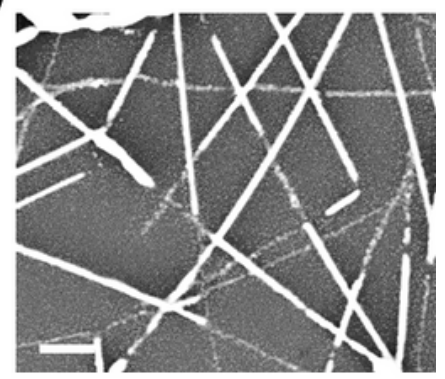

(k)

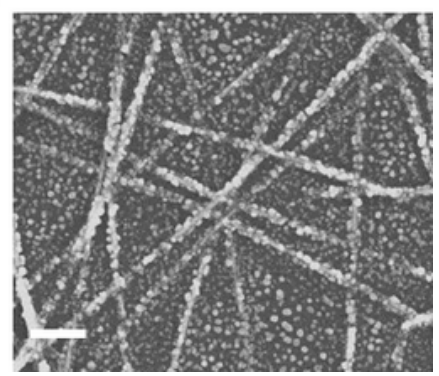

Figure 2

a Schematic illustration of the fragment formed in Chi-AsA/AgNW film and the conductivity inspection by dual-probe ohmmeter. $b$ Infrared images of the degradation process under $200 \mathrm{~mA} \mathrm{~cm}-1$ at $45 \%$, 95\%, $97 \%$ and $100 \%$ of the lifetime, respectively. c-k Optical microscope and SEM images of the failed 
samples: Chi-AsA/AgNW composite film at $200 \mathrm{~mA} \mathrm{~cm}-1$ (c, d, e), pristine AgNW film at $200 \mathrm{~mA} \mathrm{~cm}-1$ (f, g, h) and pristine AgNW film at $100 \mathrm{~mA} \mathrm{~cm}-1(\mathrm{i}, \mathrm{j}, \mathrm{k})$. SEM images demonstrate the preserved conductive area $(d, g, j)$ and the crack area $(e, h, k)$. The bar in $c, f, i$ is $60 \mu \mathrm{m}$, in $d-e, g-h, j-k$ is $300 \mathrm{~nm}$.

(a)

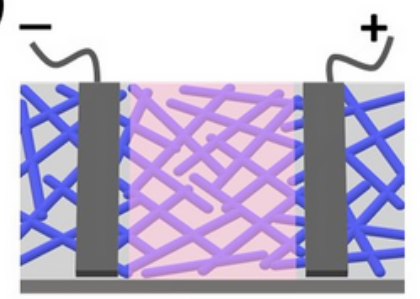

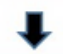

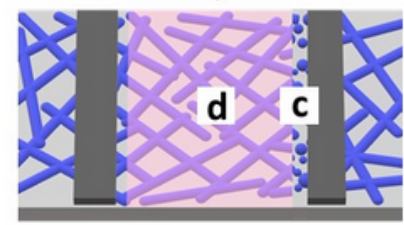

I

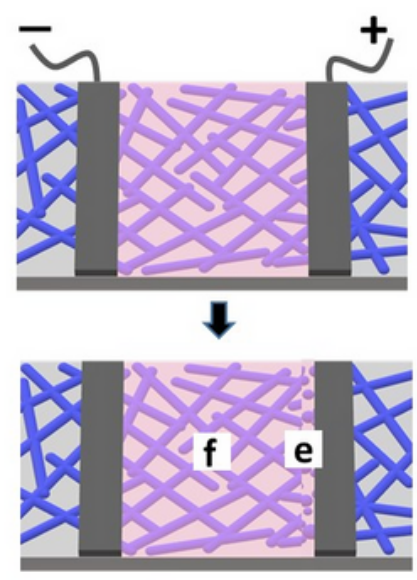

II

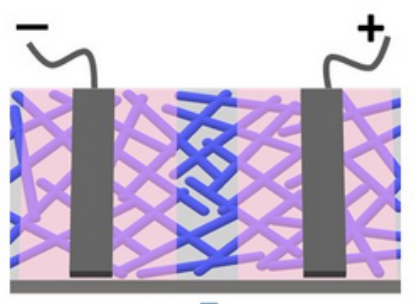

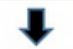

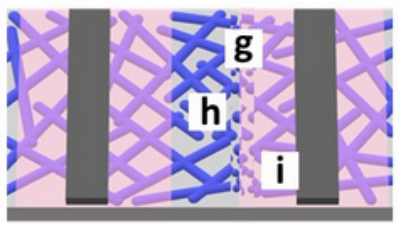

III (b)

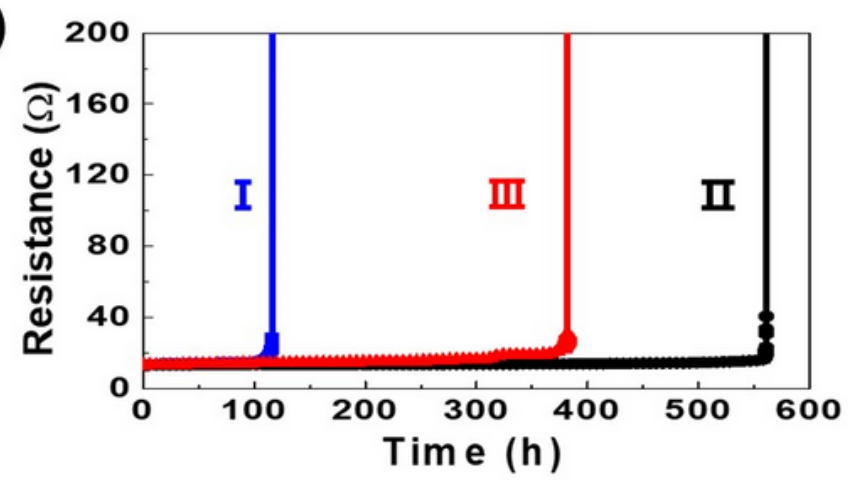

(c)

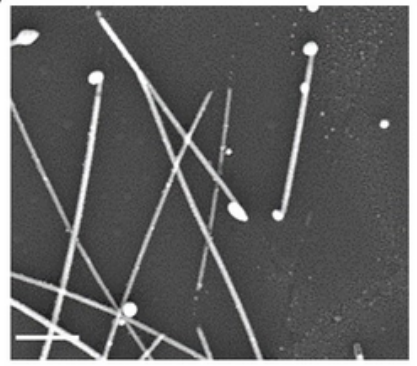

(d)

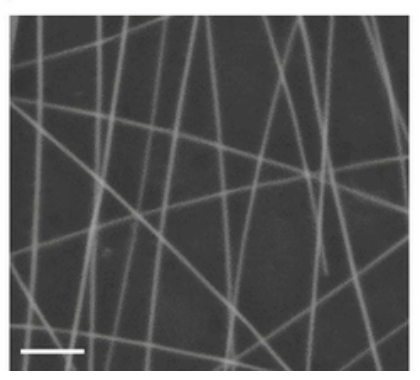

(e)

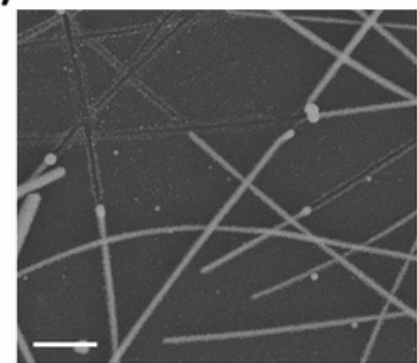

(f)

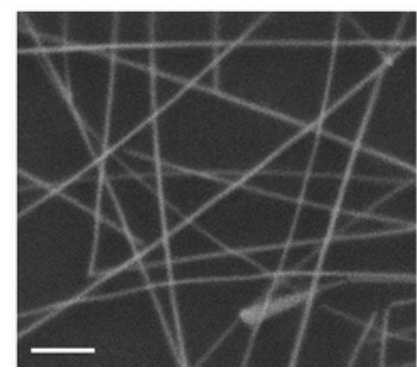

(g)

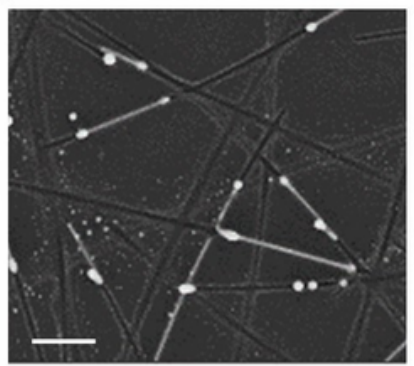

(h)

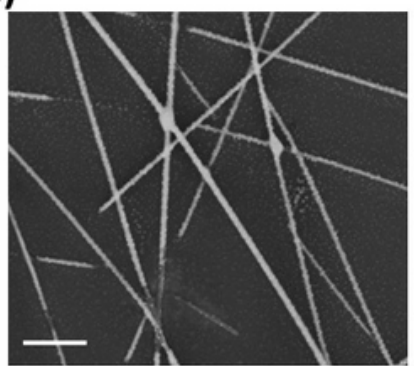

(i)

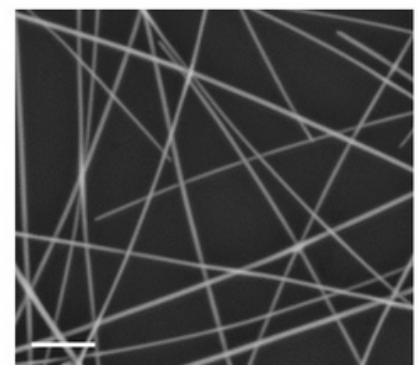

Figure 3

a Schematic of AgNW networks with partial Chi-AsA coating area and $b$ the corresponding resistancetime curve. c-i SEM images corresponding to the sites in a: the pristine region near positive connection 
electrode (c) and the Chi-AsA covered region in the middle (d) of Sample l; the boundary region between Chi-AsA coating and positive connection electrode (e) and the Chi-AsA covered region in the middle (f) of Sample II; the boundary region between Chi-AsA coating and middle pristine network (g), the middle pristine region (h) and the Chi-AsA covered region (i) of Sample III. The bar is $400 \mathrm{~nm}$.

(a)

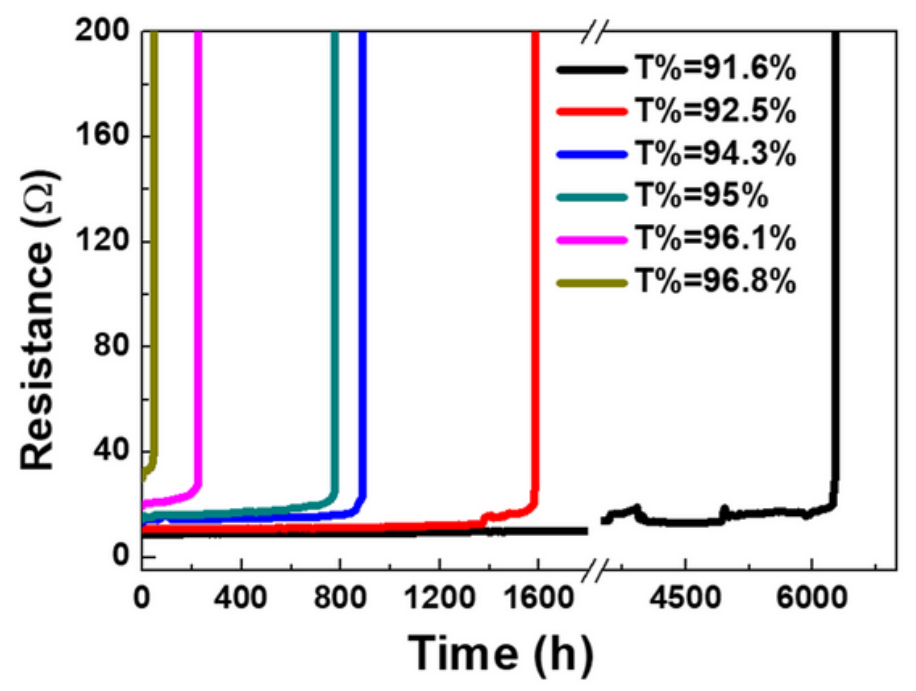

(c)

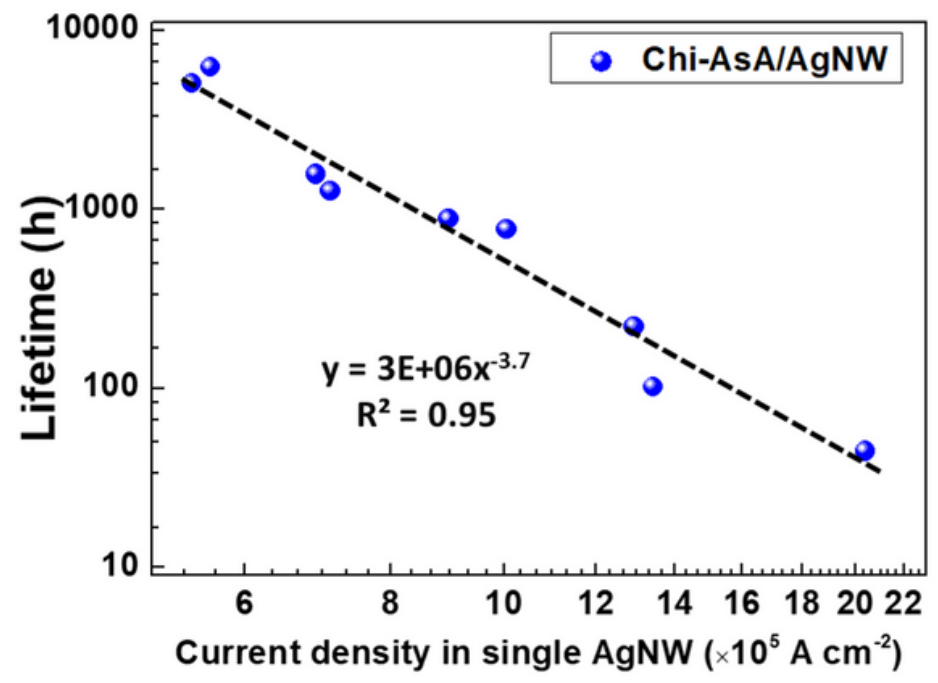

(b)

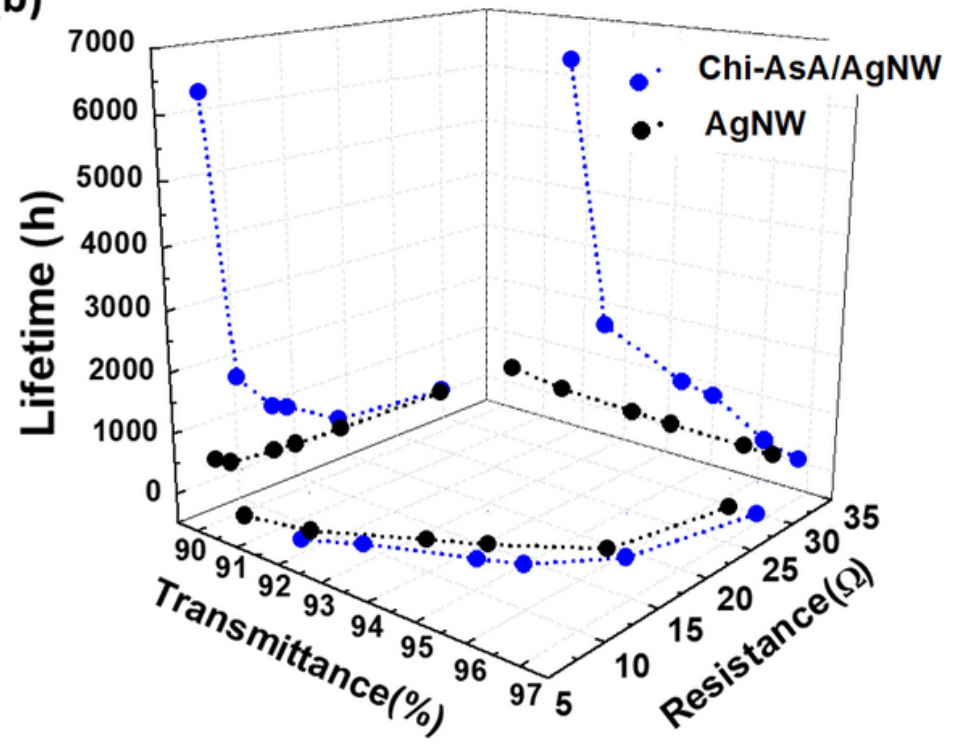

(d)

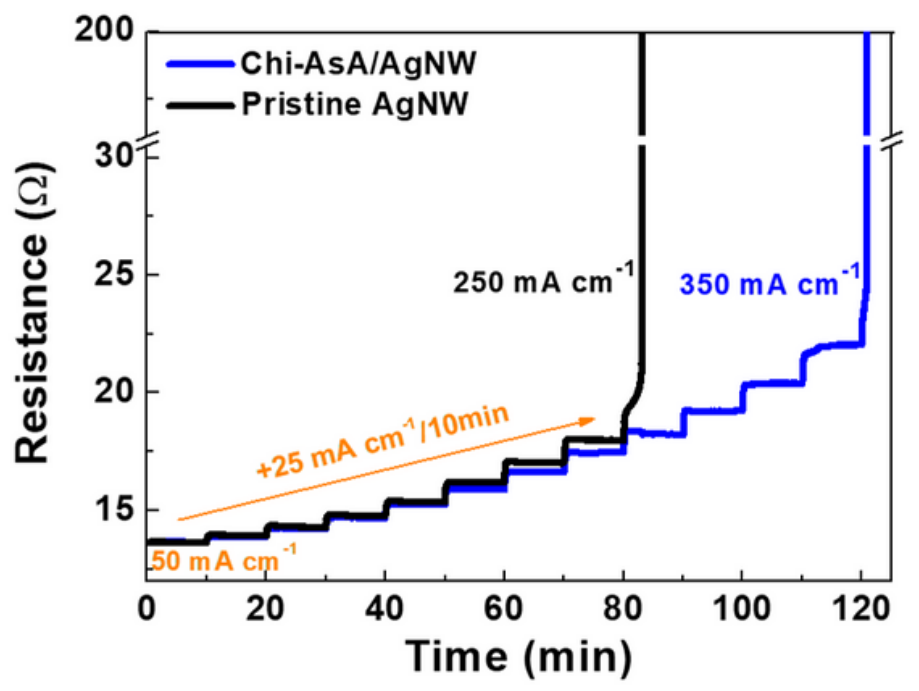

Figure 4

a Resistance-time curve for Chi-AsA/AgNW composite films with different AgNW deposition densities at $200 \mathrm{~mA} \mathrm{~cm}-1$. b 3D diagram of the relationship among lifetime at $200 \mathrm{~mA} \mathrm{~cm}-1$, transmittance and initial resistance of Chi-AsA/AgNW and AgNW films. c Lifetime of the Chi-AsA/AgNW films at $200 \mathrm{~mA} \mathrm{~cm}-1$ as a function of current density in single AgNW. d Resistance of Chi-AsA/AgNW and AgNW films at stepwisely increasing current density, respectively, which started at $50 \mathrm{~mA} \mathrm{~cm}-1$ and increased by $25 \mathrm{~mA} \mathrm{~cm}-1$ every 10 min. 
(a)

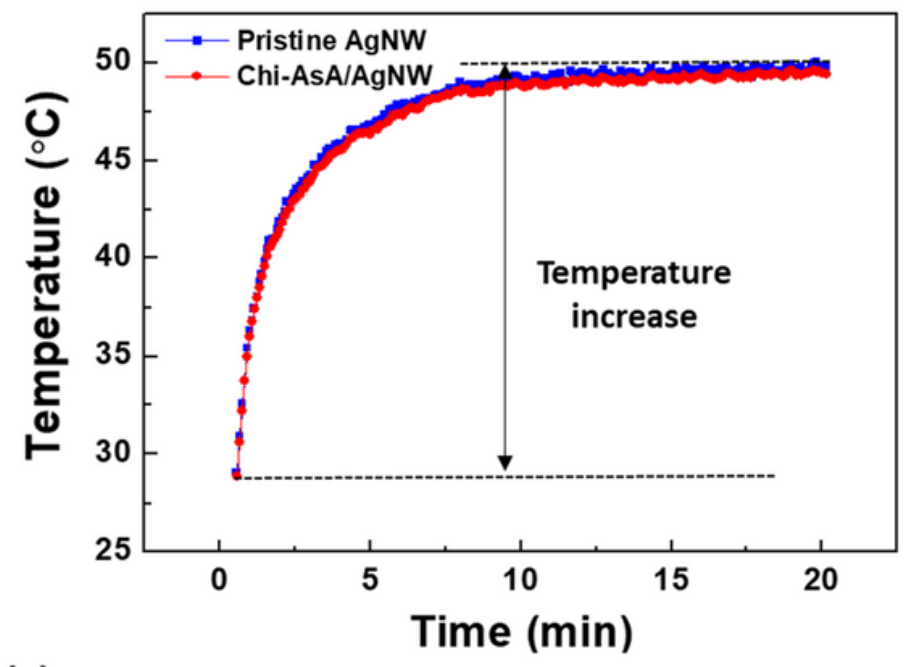

(c)

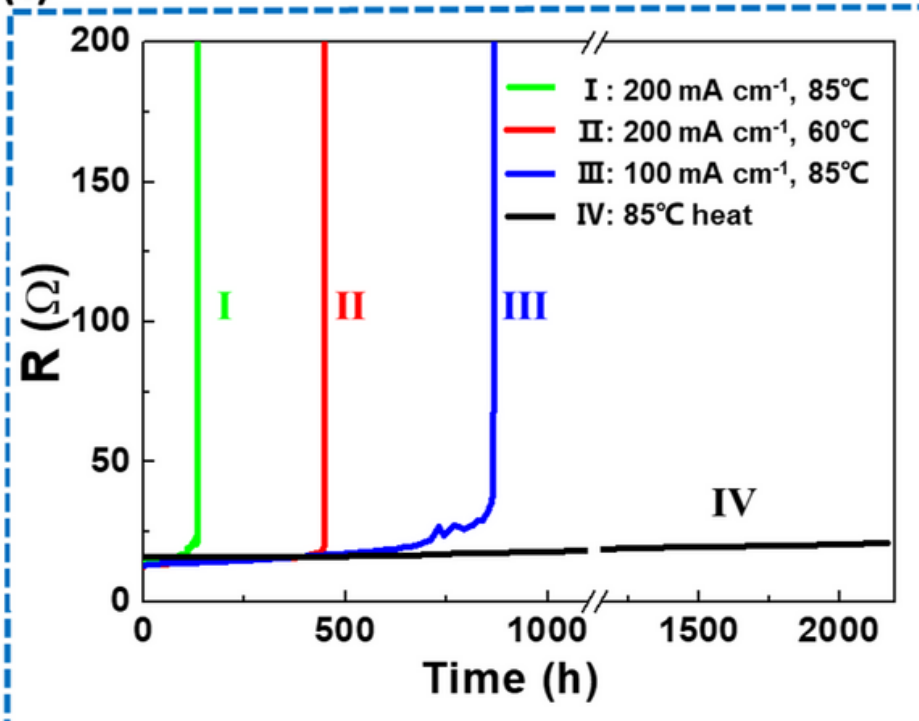

(b)

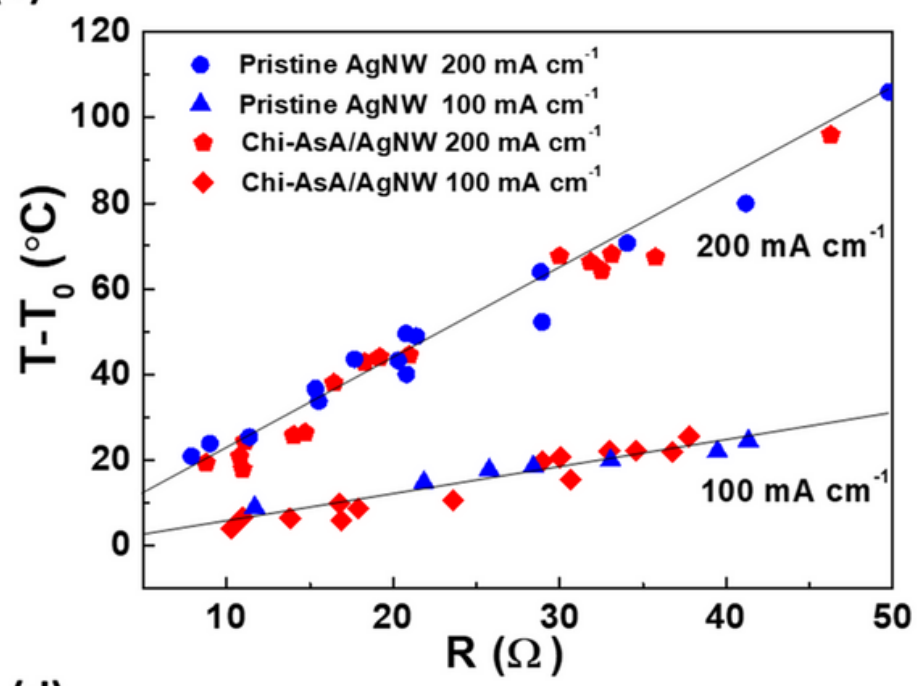

(d)

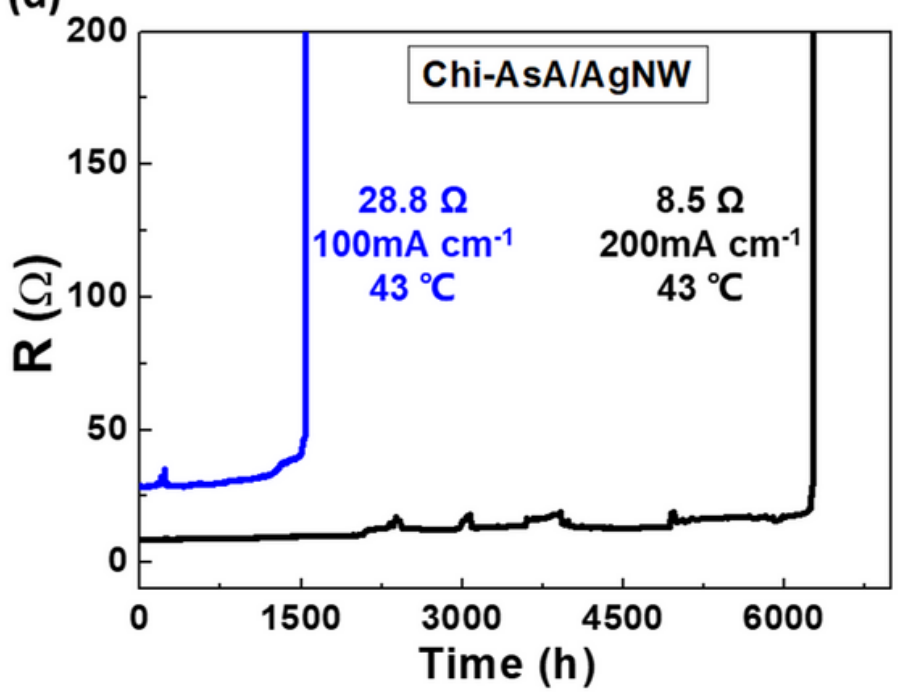

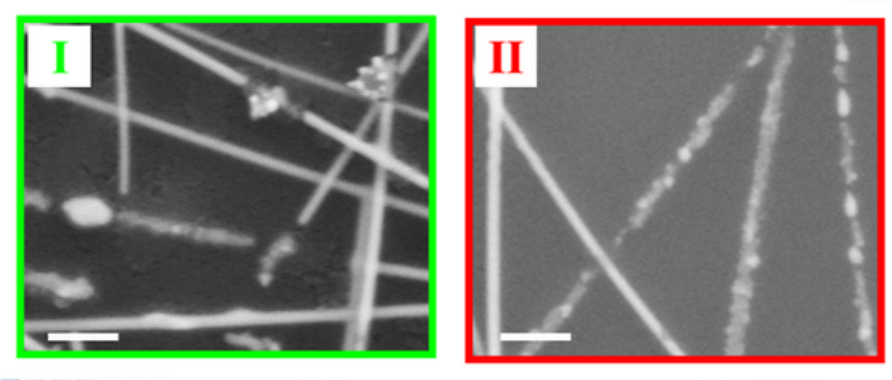
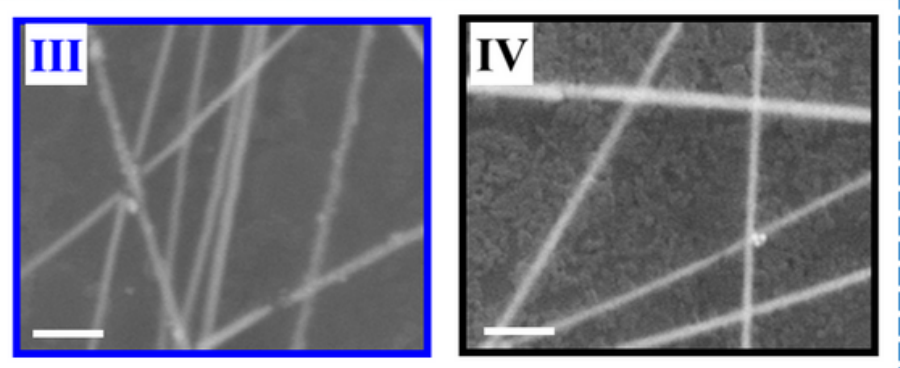

Figure 5

a Time-dependent temperature profile of pristine AgNW film and Chi-AsA/AgNW composite film applied with $200 \mathrm{~mA} \mathrm{~cm}-1$. b Temperature increase due to Joule heating as a function of initial resistance for AgNW and Chi-AsA/AgNW films at $100 \mathrm{~mA} \mathrm{~cm}-1$ and $200 \mathrm{~mA} \mathrm{~cm}-1$, respectively. c Resistance-time curves and corresponding SEM images for Chi-AsA/AgNW composite films under current and external hot plate.

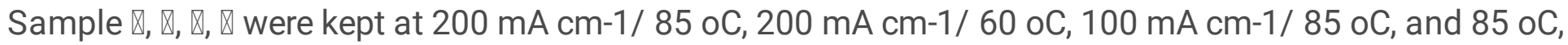
respectively. The bar in SEM is $200 \mathrm{~nm}$. d Resistance-time curves for Chi-AsA/AgNW films of $8.5 \Omega$ at 200 
mA cm-1 and $28.8 \Omega$ at $100 \mathrm{~mA} \mathrm{cm-1,} \mathrm{respectively.} \mathrm{The} \mathrm{macroscopic} \mathrm{temperatures} \mathrm{were} \mathrm{both} \mathrm{about} 43$ oC due to Joule heating.
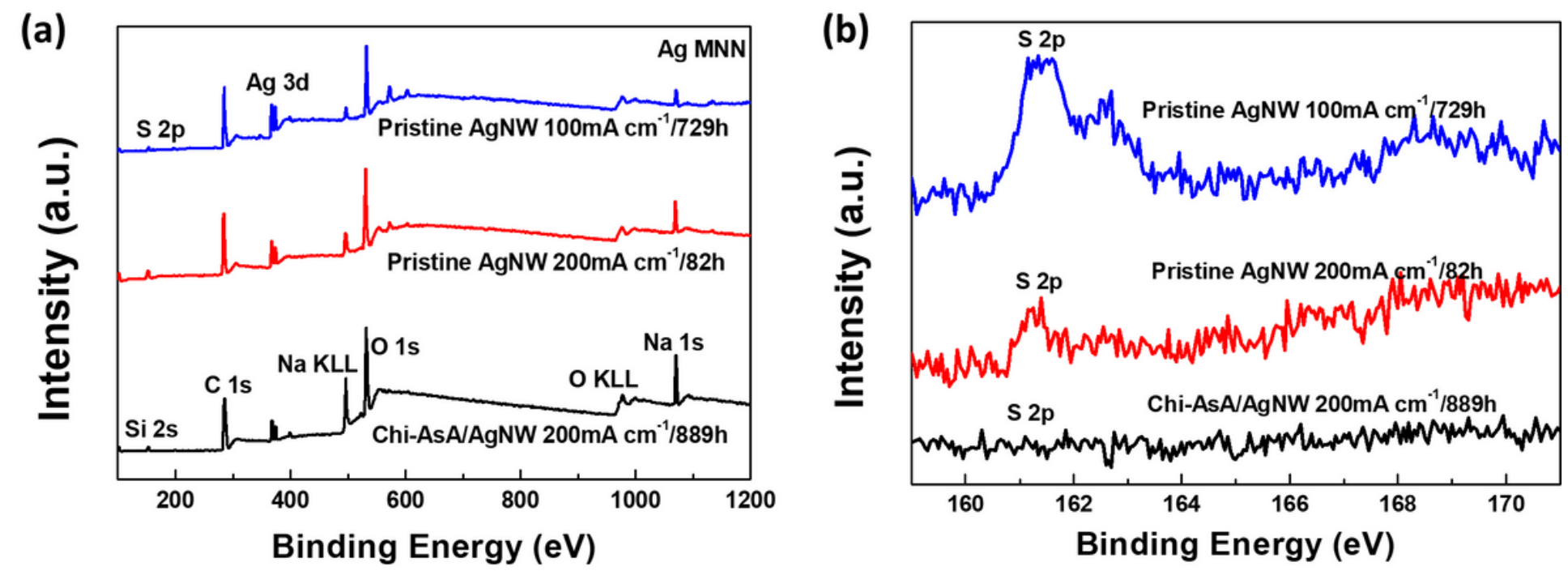

Figure 6

a Wide scan and b corresponding S 2p XPS spectra of the pristine AgNW film and Chi-AsA/AgNW composite film failed under different electrical conditions.

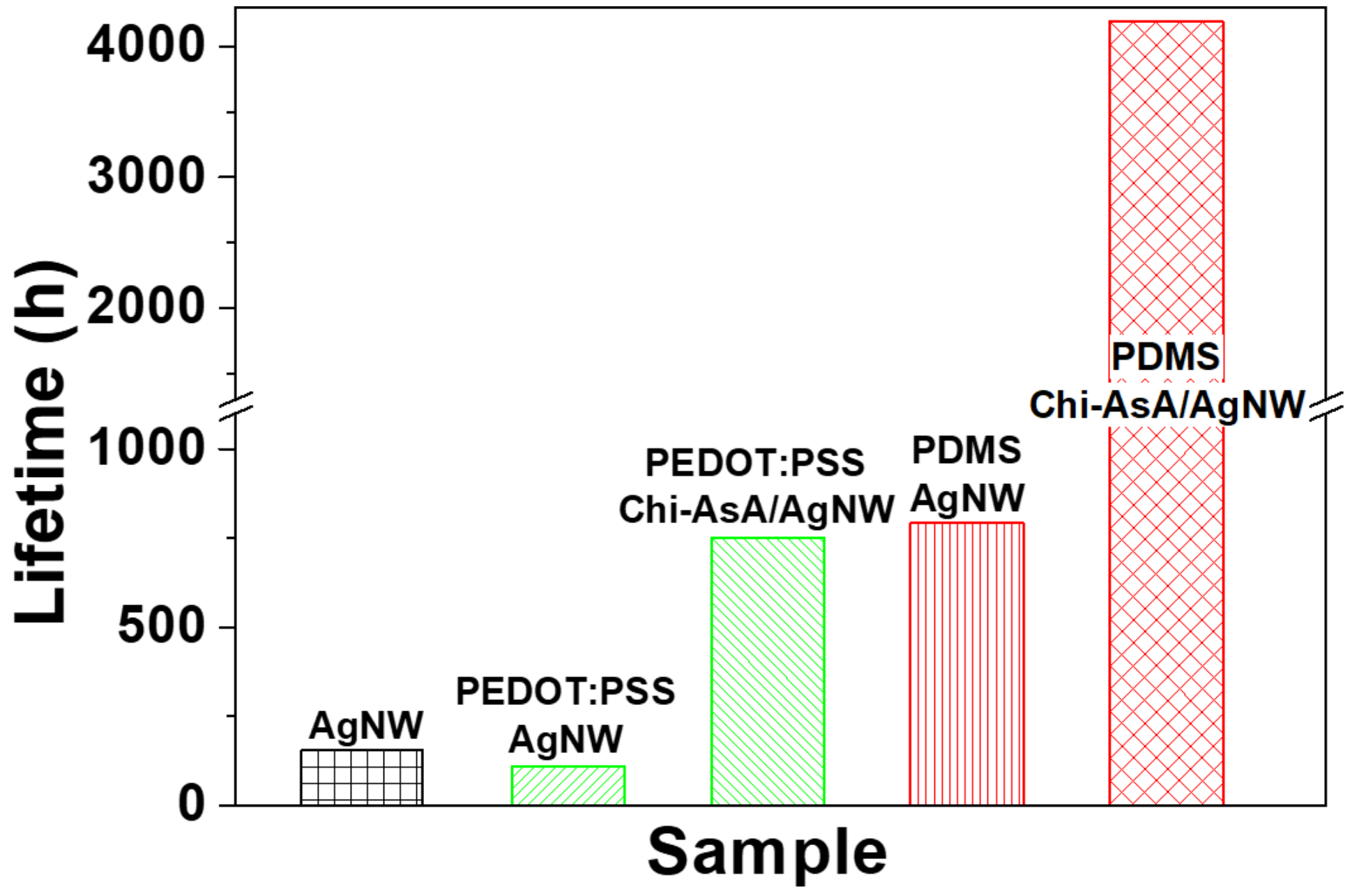

Figure 7 
Lifetime of the AgNW film and Chi-AsA/AgNW film coated with PEDOT:PSS and PDMS under $200 \mathrm{~mA} \mathrm{~cm}$ 1

\section{Supplementary Files}

This is a list of supplementary files associated with this preprint. Click to download.

- Table1.xlsx

- ESIrevision.docx 